\title{
PROGRESIÓN BIOLÓGICA Y CLÍNICA DEL CÁNCER DE PRÓSTATA INCIDENTAL (ESTADÍO Tla)
}

\author{
A.V. NÚÑEZ LÓPEZ, A. OJEA CALVO, F. DOMÍNGUEZ FREIRE, \\ A.E. ALONSO RODRIGO, B. RODRÍGUEZ IGLESIAS, J. BENAVENTE DELGADO, \\ J.M. BARROS RODRÍGUEZ
}

Servicio de Urología. Hospital Universitario Xeral-Cíes. Vigo (Pontevedra).

Actas Urol Esp. 27 (5): 345-349, 2003

\section{RESUMEN}

"PROGRESIÓN BIOLÓGICA Y CLÍNICA DEL CÁNCER DE PRÓSTATA INCIDENTAL (ESTADIO Tla)"

OBJETIVO: Analizar el comportamiento biológico y clínico del cáncer de próstata estadío Tla.

MATERIAL Y MÉTODOS: Estudio retrospectivo de 44 pacientes diagnosticados de adenocarcinoma de próstata Tla entre 1985 y 2001 . Valoramos progresión biológica y clínica, tiempo hasta la progresión, mortalidad por causa tumoral y supervivencia, con la siguiente estratificación: pacientes sin tratamiento inicial y pacientes tratados mediante radioterapia externa o prostatectomía radical.

RESULTADOS: De los 44 pacientes se observó progresión biológica en $5(11,36 \%)$ y progresión clínica en 4 (9,09\%). La mortalidad a 5 años por causa tumoral fue de 2 (4,54\%). De los 38 pacientes sin tratamiento inicial se observó progresión biológica en $5(13,15 \%)$, en un tiempo medio de 25,8 meses y progresión clínica en 4 (10,52\%), en un tiempo medio de 34,5 meses. La mortalidad a 5 años fue de $2(5,26 \%)$. En los 6 pacientes a los que se les realizó tratamiento radical no se observó progresión y viven todos. No hay diferencias estadísticamente significativas entre los dos grupos de pacientes $(\mathrm{p}=\mathrm{NS})$.

CONCLUSIONES: La progresión biológica y clínica del cáncer de próstata Tla es baja, 11,36\% y 9,09\%, respectivamente. La mortalidad a 5 años es del $4,54 \%$. No existen diferencias de supervivencia, estadísticamente significativas, entre tratados y no tratados.

PALABRAS CLAVE: Cáncer de próstata. Cáncer incidental. Progresión. Supervivencia

\section{ABSTRACT}

"CLINICAL AND BIOLOGICAL PROGRESION OF INCIDENTAL PROSTATIC CANCER (STAGE T1a)"

OBJETIVES: To analyze the biological and clinical progression of the prostate cancer stage Tla.

MATERIAL AND METHODS: Retrospective study of 44 patients diagnosed of Tla prostate carcinoma between 1985 and 2001. We value biological and clinical progression, time up to the progression, mortality for tumour reason and survival, with the following stratification: patients without initial treatment and patients treated by means of external radiotherapy or radical prostatectomía.

RESULTS: Of all 44 patients biological progression was observed in 5 (11.36\%) and clinical progression in 4 (9.09\%). The mortality to 5 years for tumour reason was of $2(4.54 \%)$. Of all 38 patients without initial treatment biological progression was observed in 5 (13.15\%), in an average time of 25.8 months and clinical progression in 4 (10.52\%), in an average time of 34.5 months. The mortality to 5 years was of 2 (5.26\%). In all 6 patients to whom radical treatment carried out them progression was not observed and they all live. There are no statistically significant differences between both groups of patients ( $p=N S)$.

CONCLUSIONS: The biological and clinical progression of the Tla prostate cancer is low, $11.36 \%$ and $9.09 \%$, respectively. The mortality to 5 years is of $4.54 \%$. Differences of survival do not exist, statistically significant, between treated and not trated.

KEY WORDS: Prostate cancer. Incidental cancer. Progression. Survival. 
$\mathrm{E}^{1}$ cáncer de próstata en estadío Tla, según la clasificación TNM de la UICC de 1997, se corresponde con un tumor clínicamente no evidente, no palpable en el tacto rectal y no visible mediante técnicas de imagen, que se detecta como hallazgo incidental en cirugía por patología prostática benigna y que afecta a un $5 \%$ o menos del tejido resecado ${ }^{1}$.

El manejo de los pacientes con cáncer de próstata en estadío Tla es todavía hoy un problema, ya que su potencial biológico varía de unos individuos a otros. Las publicaciones revisadas demuestran que es difícil identificar factores pronósticos potencialmente útiles para tomar decisiones terapéuticas ${ }^{2}$. La prostatectomía radical probablemente suponga sobretratamiento en un porcentaje importante de pacientes, mientras que la observación y seguimiento conlleva un riesgo de progresión que no podemos predecir ${ }^{3}$.

El objetivo de este estudio es aportar nuestra experiencia en relación con el comportamiento biológico y clínico del cáncer de próstata en estadio Tla.

\section{MATERIAL Y MÉTODOS}

Se revisaron las historias clínicas de 44 pacientes, en el periodo comprendido entre 1985 y 2001, diagnosticados de adenocarcinoma de próstata estadio Tla, reclasificados según la clasificación TNM de la UICC de 1997. 25 pacientes fueron diagnosticados después de una resección transuretral (RTU) de próstata por Hipertrofia Prostática Benigna y 19 pacientes después de adenomectomía.

El Gleason variaba entre 2 y 7 , siendo el valor más frecuente el de 4 (18 pacientes). El PSA oscilaba entre 0.13 y 4 , con un valor medio de 2,49 $\mathrm{ng} / \mathrm{ml}$. La edad media de los pacientes, en el momento del diagnóstico, era de 70 años (51-91 años).

El protocolo de tratamiento de los cánceres de próstata en estadio Tla varía en relación con el periodo de tiempo analizado. Entre 1985 y 1992, a los pacientes con tumores Tla con una probabilidad de supervivencia superior a 10 años se les indicaba radioterapia externa y con los pacientes con una probabilidad de supervivencia inferior a 10 años se optaba por la observación y seguimiento. Entre 1992 y 2001, a los pacientes con tumores Tla con probabilidad de supervivencia superior a 10 años se les practicaba prostatectomía radical y con los pacientes con probabilidad de supervivencia inferior a 10 años se optaba por la observación y seguimiento.

Siguiendo este protocolo, se practicó prostatectomía radical en 4 pacientes y se administró radioterapia externa en 2 pacientes. En 38 pacientes, la actitud fue expectante. El tiempo medio de seguimiento fue de 69.36 meses (18 meses-16 años).

En una de las piezas de prostatectomía no se observó tumor y se clasificó como pTO. En las otras tres piezas de prostatectomía se observó tumor en un lóbulo prostático y se reclasificaron como pT2a.

Para el control de los pacientes, según el periodo analizado, se establecieron determinaciones de fosfatasa ácida prostática, determinaciones de antígeno prostático específico (PSA) y tacto rectal cada 6 meses. En caso de progresión biológica (elevación de un 50\% de los marcadores en, al menos, dos determinaciones) y progresión clínica, se completaba el estudio diagnóstico con ecografía y gammagrafía ósea. En los casos en que se detectó progresión biológica o clínica se pautaba bloqueo androgénico completo.

Valoramos la progresión biológica y clínica, el tiempo hasta la progresión biológica, el tiempo hasta la progresión clínica y la supervivencia. Establecemos la siguiente estratificación: pacientes sin tratamiento inicial y pacientes tratados mediante prostatectomía radical o radioterapia externa.

Calculamos las curvas de supervivencia por el método de Kaplan-Meier y el grado de significación estadística (p) mediante el test de MantelHanszel.

\section{RESULTADOS}

De los 44 pacientes analizados, se observó progresión biológica en $5(11,36 \%)$ y progresión clínica en 4 (9,09\%). El tiempo medio hasta la progresión biológica fue de 25,8 meses (9-48 meses) y hasta la progresión clínica de 34,5 meses (30-48 meses). La mortalidad a 5 años, por causa tumoral, fue de 2 pacientes $(4,54 \%$ ) (Tabla I y Fig. 1). 


\section{TABLA I}

LA PROGRESIÓN BIOLÓGICA, PROGRESIÓN CLÍNICA Y MORTALIDAD EN EL CÁNCER DE PRÓSTATA T1a ES BAJA, SIN QUE SE OBSERVEN DIFERENCIAS ESTADÍSTICAMENTE SIGNIFICATIVAS ENTRE LOS PACIENTES TRATADOS CON RADIOTERAPIA O PROSTATECTOMÍA RADICAL Y LOS PACIENTES SIN TRATAMIENTO INICIAL (m: meses; a: años)

\begin{tabular}{|c|c|c|c|c|c|c|}
\hline & $\begin{array}{l}\text { Número de } \\
\text { pacientes }\end{array}$ & $\begin{array}{l}\text { Progresión } \\
\text { biológica }\end{array}$ & $\begin{array}{c}\text { Tiempo hasta } \\
\text { progresión } \\
\text { biológica }\end{array}$ & $\begin{array}{l}\text { Progresión } \\
\text { clínica }\end{array}$ & $\begin{array}{l}\text { Tiempo hasta } \\
\text { progresión } \\
\text { clínica }\end{array}$ & $\begin{array}{l}\text { Mortalidad a } \\
5 \text { años }\end{array}$ \\
\hline Sin tratamiento & 38 & $5(13,15 \%)$ & $\begin{array}{l}25,8 \text { meses } \\
(9 \mathrm{~m}-4 \mathrm{a})\end{array}$ & $4(10,52 \%)$ & $\begin{array}{c}34,5 \text { meses } \\
(30 \mathrm{~m}-4 \mathrm{a})\end{array}$ & $2(5,26 \%)$ \\
\hline $\begin{array}{l}\text { Prostatectomía } \\
\text { o radioterapia }\end{array}$ & 6 & No & & No & & Viven todos \\
\hline Total & 44 & $5(11,36 \%)$ & $\begin{array}{c}25,8 \text { meses } \\
(9 \mathrm{~m}-4 \mathrm{a})\end{array}$ & $4(9,09 \%)$ & $\begin{array}{c}34,5 \text { meses } \\
(30 \mathrm{~m}-4 \mathrm{a})\end{array}$ & $2(4,54 \%)$ \\
\hline
\end{tabular}

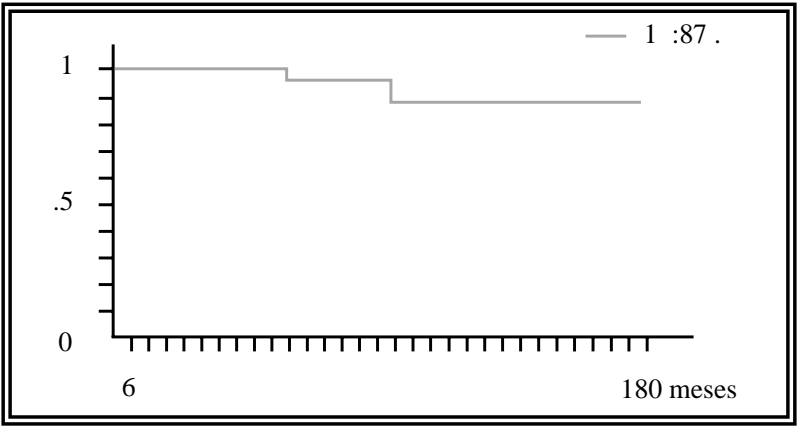

FIGURA 1. Curva de supervivencia de Kaplan-Meier para el total de pacientes con cáncer de próstata estadio T1a. La probabilidad de supervivencia global de los pacientes con cáncer de próstata T1a es del $87 \%$ a los 180 meses.

De los 38 pacientes sin tratamiento inicial, se observó progresión biológica en $5(13,15 \%)$ y progresión clínica en $4(10,52 \%)$. El tiempo medio hasta la progresión biológica fue de 25,8 meses (9-48 meses) y hasta la progresión clínica de 34,5 meses (30-48 meses).La mortalidad a 5 años, por causa tumoral, fue de 2 pacientes $(5,26 \%)$ (Tabla I y Fig. 2).

En los 4 pacientes a los que se realizó prostatectomía radical y en los 2 pacientes a los que se administró radioterapia externa con intención curativa no se observó progresión y viven todos (Tabla I).

La supervivencia a 180 meses es de un $87 \%$ para el total de pacientes, de un $100 \%$ para el grupo de pacientes con tratamiento radical y de un $84 \%$ para los pacientes sin tratamiento inicial (Figs. 1 y 2).

Las diferencias, en cuanto a supervivencia, entre el grupo de pacientes tratados y no tratados no son estadísticamente significativas $(p=N S)$.

\section{DISCUSION}

El manejo de los pacientes con cáncer de próstata incidental (estadio Tla) es todavía un capítulo controvertido. Würnschimmel y cols. ${ }^{3}$ en el 92, opinan que estos pacientes, si son jóvenes y con buena expectativa de vida, son subsidiarios de prostatectomía radical. En su trabajo, en los pacientes en los que se decide una actitud expectante ("esperar y ver"), el tumor progresó en un $23,4 \%$ de los casos. En el 50\% de los pacientes a los que se realizó prostatectomía radical, la pieza quirúrgica se correspondía con un mayor estadio anatomopatológico, mientras que, en un 30\% de los casos la pieza de prostatectomía estaba libre de tumor (sobretratamiento).

Leisinger y cols. ${ }^{2}$ en el 94, revisaron los trabajos publicados hasta ese momento observando que los tumores Tla no tratados progresaban en un 16 a 36\% de los casos, dependiendo del tra-

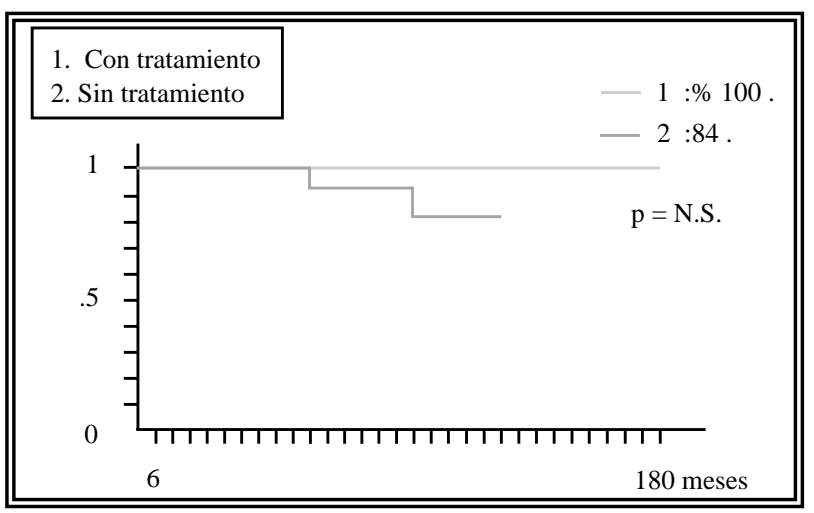

FIGURA 2. Curvas de supervivencia de Kaplan-Meier para el grupo de pacientes con tratamiento radical, prostatectomia o radioterapia1 y para el grupo de pacientes sin tratamiento inicial2. La probabilidad de supervivencia es del $100 \%$ para los pacientes tratados frente al $84 \%$ para los pacientes no tratados, aunque sin diferencias estadisticamente significativas ( $p=N S$ ). 
bajo revisado. En las piezas de prostatectomía radical de los cánceres Tla se encontró tumor residual, después de RTU de próstata, en un 48 a $83 \%$ de los casos. Según sus observaciones, la prostatectomía radical debería ser el tratamiento de elección en pacientes jóvenes con tumores Tla. También encontraron una correlación entre progresión y edad del paciente, volumen de tumor y diferenciación histológica. En este sentido, la RTU de próstata no permite establecer el volumen total de tumor, que es un factor pronóstico importante.

Steinberg y cols. ${ }^{4}$ en el 98 y Berner y cols. ${ }^{5}$ en el 99, concluyen que "esperar vigilante" es la mejor opción en tumores bien y moderadamente diferenciados, de poco volumen y en pacientes con expectativa de vida $<10$ años. Esta opción no se debería aplicar a hombres sanos con una expectativa de vida $>10$ años o tumores agresivos y pobremente diferenciados. Estos pacientes debería recibir tratamiento con intención curativa.

En los últimos años la actitud con este cáncer tiende a ser más conservadora. Así, Schmid y cols. ${ }^{6}$ en el año 2001, definen el concepto de "monitorización activa" mediante PSA, TRUS y biopsia de la próstata residual, que se podría indicar a pacientes con tumores en estadio Tla bien-moderadamente diferenciados y expectativa de vida $>10$ años. También, estaría indicado en pacientes con tumores en estadio T1b-T2b bienmoderadamente diferenciados y expectativa de vida $<10$ años.

Más recientemente, Carter, Walsh y cols. ${ }^{7}$ opinan que el manejo expectante incluso puede ser una buena alternativa en hombres mayores con tumores Tlc de pequeño volumen. En su trabajo observaron que estos tumores progresaban en un $31 \%$ de los casos y que los pacientes sometidos a prostatectomía radical presentaban tumores organoconfinados en un $92 \%$ de los casos.

En el análisis de los resultados de nuestro estudio, aunque es un grupo de pacientes muy heterogéneo, en cuanto a los métodos de diagnóstico, tratamiento y control de los que se disponían según el periodo de tiempo analizado, observamos en términos generales un buen comportamiento de los cánceres de próstata Tla con un riesgo de progresión bajo.
En los pacientes en los que la actitud fue radical, mediante prostatectomía o radioterapia, no hubo progresión biológica ni clínica. Sin embargo, no hemos podido demostrar diferencias estadísticamente significativas cuando se compararon los resultados con los pacientes en los que la actitud fue expectante. Además, en una de las piezas de prostatectomía no se observó tumor residual, lo que supone sobretratamiento en $25 \%$ de los casos en los que se hizo prostatectomía radical.

Actualmente, se intenta definir factores de progresión como la expresión anormal de $\mathrm{p} 53^{8}$, la aneuploidía $^{2}$ o la pérdida de expresión del gen NKX3. $1^{9}$, que nos permitan identificar aquellos pacientes que puedan beneficiarse de un tratamiento radical. En este sentido, Cheng y cols. ${ }^{10}$ estudian pacientes diagnosticados de adenocarcinoma de próstata Tla tras RTU de próstata, encontrando una asociación estadísticamente significativa entre cantidad de tejido resecado y progresión tumoral. No encuentran asociación estadísticamente significativa entre progresión y grado Gleason, volumen tumoral, número de focos tumorales o presencia de neoplasia intraepitelial prostática de alto grado. Estos autores concluyen que, pacientes con tejido resecado mayor o igual a $30 \mathrm{~g}$ tienen un pronóstico excelente y podrían ser manejados de forma conservadora.

\section{CONCLUSIONES}

La progresión biológica y clínica del cáncer de próstata Tla es baja, 11,36\% y 9,09\%, respectivamente. La mortalidad a 5 años, por causa tumoral, es de un 4,54\%. Las diferencias de supervivencia, entre pacientes sin tratamiento inicial y pacientes tratados mediante prostatectomía radical o radioterapia externa, no son estadísticamente significativas.

En vista de los resultados y análisis de la literatura, no nos podemos decantar por una actitud radical o conservadora. Están pendientes estudios sobre factores pronósticos que permitan identificar aquellos pacientes con alto riesgo de progresión tumoral y que se beneficiarian de un tratamiento radical, frente a los pacientes de bajo riesgo en los que estaría indicado tratamiento conservador. 


\section{REFERENCIAS}

1. HERMANEK P, HUTTER RVP, SOBIN LH, WAGNER G, WITTEKIND CH (eds): TNM atlas. Ilustratec guide of the TNM/pTNM classification of malignant tumours. Springer-Verlag. Berlin 1997. Edición 1997 (4모): 263-326.

2. LEISINGER HJ.: Prostatic cancer stage T1. "incidental carcinoma". Review of the literature and critical reappraisal of the classification system. Ann Urol 1994; 28 (4): 229-234.

3. WÜRNSCHIMMEL E, LIPSKY H: Incidental carcinoma of the prostate- "wait and see" or radical prostatectomy?. Urologe-A 1992; 31 (1): 48-51.

4. STEINBERG GD, BALES GT, BRENDLER CB.: An analysis of watchful waiting for clinically localized prostate cancer. $J$ Urol 1998; 159 (5): 1431-1436.

5. BERNER A, HARVEI S, SKJORTEN FJ.: Follow-up of localized prostate cancer, with emphasis on previous undiagnosed incidental cancer. BJU Int 1999; 83 (1): 47-52.

6. SCHMID HP, ADOLFSSON J, AUS G.: Active monitoring (deferred treatment or watchful waiting) in the treatment of prostate cancer. Eur Urol 2001; 40 (5): 488-494.
7. CARTER HB, WALSH PC, LANDIS P, EPSTEIN JI.: Expectant management of nonpalpable prostate cancer with curative intent: preliminary results. $J$ Urol 2002; 167 (3): 1231-1234.

8. VAN-VELDHUIZEN PJ, SADASIVAN R, CHERIAN R, DWYER T, STEPHENS RL.: p53 expression in incidental prostatic cancer. Am J Med Sci 1993; 305 (5): 275-279.

9. BOWEN C, BUBENDORF L, VOELLER HJ et al.: Loss of NKX3.1 expression in human prostate cancers correlates with tumor progression. Cancer Res 2000; 60 (21): 6111-6115.

10. CHENG L, BERGSTRALH EJ, SCHERER BG et al.: Predictors of cancer progression in Tla prostate adenocarcinoma. Cancer 1999 mar 15; 85 (6): 1300-1304.

Dr. A.V. Núñez López

Servicio de Urología. Hosp. Univ. Xeral-Cíes

C/ Pizarro, 22

36204 Vigo (Pontevedra)

(Trabajo recibido el 3 septiembre de 2002) 